\title{
Traffic Adaptive Geographic Routing for Vehicular Ad Hoc Networks
}

\author{
Xiang-yu Bai \\ College of Computer Science \\ Inner Mongolia University \\ Hohhot, China \\ baixiangyu@imu.edu.cn, \\ Xin-ming Ye \\ College of Computer Science \\ Inner Mongolia University \\ Hohhot, China \\ xmy@imu.edu.cn
}

\author{
Yan-jun Huang \\ College of Computer Science \\ Inner Mongolia University \\ Hohhot, China
}

Jun Li

Institute of Computing Technology

Chinese Academy of Sciences Beijing, China

lijun@ict.ac.cn

\begin{abstract}
Vehicular Ad Hoc Network (VANET) has recently attracted considerable attention. The design of routing protocol for VANET is a challenge given rapid topology changes and frequent network disconnection. Current routing protocols usually use geographic information to forward packets. In this paper, we propose a traffic adaptive routing scheme called TAGR for inter-vehicle communication in urban scenarios. The key differences between TAGR and other geographic protocols are the process of real-time vehicle traffic estimation and Traffic adaptive selection of optimal route. Firstly, the position data that our location service provides is analyzed and aggregated to estimate vehicle densities of roads. Secondly, TAGR considers the movements of nodes (vehicles) and takes advantage of the predictable mobility pattern of vehicles. TAGR utilizes traffic information and movement predication to create adaptive connectivity model which improves the routing process by selecting the most stable route. Simulations in realistic city environments are conducted to compare the proposed protocol with GSR and GyTAR protocols. The results show that TAGR outperforms the others in terms of data delivery ratio and average delay.
\end{abstract}

Keywords-Vehicular Ad Hoc Network; Routing; Grographic; Traffic Adaptive

\section{INTRODUCTION}

Vehicular Ad Hoc Network (VANET) has been envisioned as a promising approach for Intelligent Transportation System (ITS). VANET consists of a collection of vehicles that are equipped with wireless communication devices, GPS, digital maps and optional sensors. Vehicles (also called nodes) dynamically set up an ad hoc network without the aid of infrastructure, and the messages (unicast, multicast, geocast and broadcast) for different purposes can be relayed to destination via multi-hop vehicle communication. Such networks are envisaged to support various functionalities, including vehicle safety, traffic efficiency, travel convenience, etc [1]. In this paper, we focus on the unicast routing for inter-

Sponsored by National Nature Science Foundation of China (NSFC NO. 61062004), Nature Science Foundation of Inner Mongolia（NO.

2010MS0901) vehicle communication in city scenarios, where two moving vehicles exchange information, such as files sharing, instant messages, back-seat games and so on.

VANET discriminates itself from conventional mobile ad hoc network (MANET) by some unique characteristics. First, the topology of VANET changes rapidly because of fast movement of vehicles. Second, network disconnections frequently happen especially in the case of low vehicle density. Third, the nodes are constrained by the layout of roads, so are the transmissions of packets. These characteristics raise the challenges of communication in VANET. Traditional ad hoc routing protocols, such as AODV, DSR, and OLSR, can not be directly applied to VANET because of high data loss rates. A number of geographic routing (also called location-based routing) protocols have been proposed. With the support of location service, e.g. GLS[2], which helps the nodes acquire destinations' positions, GPSR[3] uses the greedy forwarding method where next hops are chosen based on nodes that are geographically closer to the destination. GSR [4] takes into account the city road network and computes paths by using Dijkstra's shortest path algorithm, where greedy method is taken to forward packet along roads between intersections. Furthermore, traffic aware routing protocols, such as VADD[5], CAR[6], GyTAR[7] and RBVT[8] etc, use vehicle traffic condition to identify road-based route with high connectivity. These approaches can help to optimize route selection, but they rely on additional supports: infrastructural traffic system $[5,7]$ or broadcast-based traffic collection process [6,8]. In movement-based routing algorithm [9-11], a series of data forwarding strategies are proposed, which select routes based on the position prediction of neighbor node or forwarder nodes.

In this paper, we propose a novel VANET routing protocol called Traffic Adaptive Geographic Routing (TAGR) for intervehicle communication in city environments. TAGR combines the idea of traffic aware and movement prediction with 
geographic routing. Based on the vehicle location information supplied by our location service VLS[12], a new distributed approach of real-time traffic estimation is proposed, where the position data is utilized to compute vehicular density of each road. A traffic-based route decision method is presented, which dynamically creates paths consisting of successive intersections with high connectivity for packet delivery. Furthermore, the availability of neighbor nodes is scored with position, speed and direction, and TAGR chooses the best one to avoid link rupture so that frame loss rate is reduced while the forwarding efficiency is improved. With these two methods, TAGR can select stable route with respect to high mobility of vehicles which causes fast topology changes and frequent network segmentation.

\section{THE MODEL OF TAGR}

\section{A. Assumption}

We are considering a scenario in which a set of vehicles are moving in a city. Each vehicle is equipped with an embedded computer. A connected GPS receiver provides its capability of self-localization and synchronizing the clock in the whole network. A digital map is installed on the compute with an interval data-structure which represents the roads of a city. GPS and digital maps have been default devices in more and more vehicles, so this assumption is reasonable in near future. We also assume that for the sake of safety, all vehicles are supposed to periodically send beacon messages to inform the nodes in the vicinity of their current position, velocity and direction.

\section{B. Basic Location Service}

In our previous work, we have proposed a map-based vehicle location service protocol (VLS) [12], which may provide the foundation for traffic estimation. As shown in Fig.1, the rectangle area, which is divided into $\mathrm{M} \times \mathrm{N}$ small regions, represents the city where vehicles move around. In each region, digital map information is utilized to build the set of location server candidates and a hash-based server selection algorithm helps a node associate its ID with a candidate, i.e. determine its location server. The node updates position information to its location servers in all regions at a rate proportional to its speed, and other nodes send messages to its location server to query the position information as needed.

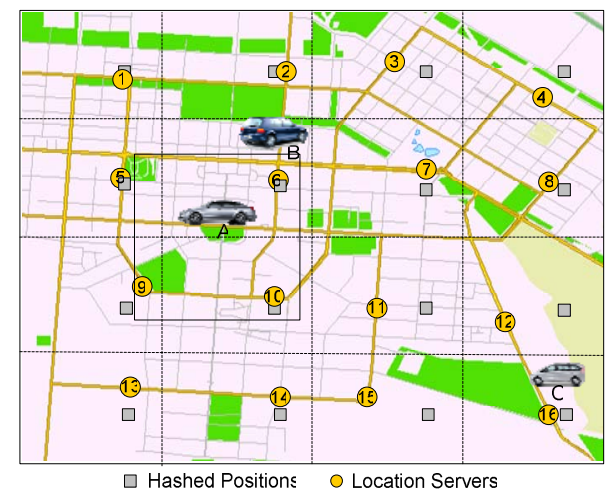

Fig 1. Location Servers in VLS

\section{Distributed Traffic Estimation}

Considering that the location servers in every region hold the position of all vehicles in the network, the position information distributed in different location servers can be collected to estimate vehicular traffic. For a given region, the location servers are organized as an MST (Minimum Spanning Tree) structure according to road layout. All location servers are un-decided initially. The servers near the center of a region start a back off timer. The deferred time $\Delta t$ is calculated as follows:

$$
\Delta t=\frac{\operatorname{distance}\left(p_{i}, p_{c}\right)}{a+b} \cdot t_{\max }
$$

Where $P_{i}$ and $P_{c}$ represent the positions of server and region center, $a$ and $b$ represent side length of the region, and $t_{\max }$ represents the maximal deferred time. When time is up, the servers send Root Advertisement (RA) packet to compete for the root the tree. If a server has heard from others it will announce itself as an offspring, otherwise it becomes root for the region. The root server decides other location servers being the branches or leaves in the MST structure according to their geographical location in the region. Then, it encapsulates the topology of tree into RA packet and broadcasts to all other servers.

For periodically estimating traffic of roads, the location information in each server is summarized into a special data structure, traffic map (RoadID, SegmentID, VehicleList), which represents the vehicle number in each road segment. At the beginning of each interval, leaf location servers encapsulate traffic map into packets and send to their parent servers. When a location server receives packets from its sub-trees, it merges its own traffic map into an aggregated traffic map, which will be forwarded to its parent accordingly. The aggregation method can effectively suppress the number of packets in the network. After attaining aggregated traffic maps from all its branches, the root starts a computing process to estimate the traffic for the whole network and distributes the result to all location servers in the region.

\section{Road-Based Route Selection}

Considering the challenges faced in urban environment, a road-based geographic routing method is proposed. The network is represented as a directed graph $G(V, E)$ with sets of vertices $V$, connected by directed edges $E$. $V$ represents the set of intersections on the road connected by the edges $E$ that represents the set of directional roads connecting these intersections. The source and destination nodes are regarded as additional vertices, each dividing a road into two edges. The weights on the edges represent the priority considering the traffic density and the vehicle speed on the road. The best edge (the edge with the lowest weight) is the road that has the highest accumulative traffic and the highest average speed. The weight $W$ is calculated as follows: 


$$
W= \begin{cases}\frac{1}{\alpha \cdot \frac{\sum_{i=1}^{m} n_{i}}{m \cdot l}+\beta \cdot \frac{|\rho|}{(k-m) \cdot l}} & 0<m<k \\ \frac{L}{\beta \cdot|V|} & m=0 \\ \frac{L}{\alpha \cdot \sum_{i=1}^{k} n_{i}} & m=k\end{cases}
$$

Where, $L$ represents the road length, $l$ represents the segment length, $k=L / l$ represents the number of segments in a road, $m$ represents the number of segments where exist vehicle(s), $|\vec{v}|$ represents the average vehicle speed in different directions, $n_{i}$ represents the vehicle number in a road segment, $\alpha$ and $\beta$ represent weighting factors for the vehicular traffic and speed respectively.

\section{E. Connectivity Adaptive Forward}

When a node uses greedy strategy to forward packets, it will rely on the position information in its neighbor table. If the neighbor vehicle nearest to the next intersection or destination moves rapidly, inaccurate positions may result in link rupture during data transmission. We propose a new strategy based on movement predication to avoid loss of packets. Firstly, the packet sender or the forwarder node computes the current position of each neighbor using the recorded history information. As shown in Fig.2, the source node (or a forwarder) $S$ and destination node (or next intersection) $D$ are located at $\left(x_{S}, y_{S}\right)$ and $\left(x_{D}, y_{D}\right)$ at time $t . S$ would transmit data to $D$ and the direction of packet forward is $\theta_{S D}$.

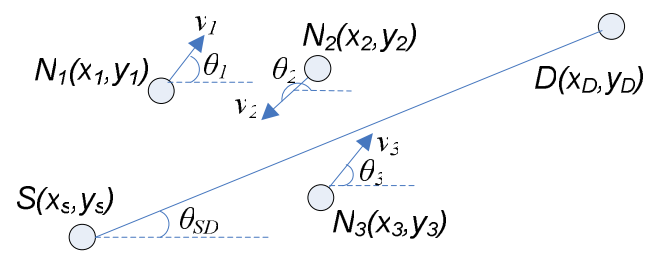

Fig 2. Movement adaptive next-hop forwarder selection

A neighbor node of $\mathrm{S}, N_{i}$, have broadcasted its beacons at time $t_{0}$ and $t_{1}$, and each beacon includes position, velocity and direction, represented as $\left(\left(x_{i 0}, y_{i 0}\right), v_{i 0}, \theta_{i}\right),\left(\left(x_{i 1}, y_{i 1}\right), v_{i l}, \theta_{j}\right)$. We assume that vehicles move along the road with constant directions $\theta_{i}$ between two intersections. The current accurate position of the node $N_{i}$ can be calculated as follows:

$$
\left\{\begin{array}{l}
x_{i}=x_{i 1}+v_{i 1} \cdot \cos \left(\theta_{i}\right) \cdot\left(t-t_{1}\right)+\frac{1}{2} \cdot \frac{v_{i 1}-v_{i 0}}{t_{1}-t_{0}} \cdot \cos \left(\theta_{i}\right) \cdot\left(t-t_{1}\right)^{2} \\
y_{i}=y_{i 1}+v_{i 1} \cdot \sin \left(\theta_{i}\right) \cdot\left(t-t_{1}\right)+\frac{1}{2} \cdot \frac{v_{11}-v_{i 0}}{t_{1}-t_{0}} \cdot \sin \left(\theta_{i}\right) \cdot\left(t-t_{1}\right)^{2}
\end{array}\right.
$$

Secondly, for the neighbors within the transmission range of node $S$, the availability of each node is scored according to: availability $\left(S, N_{i}\right)=\chi \cdot\left(1-\frac{d_{N_{i} D}}{d_{S D}}\right)+\delta \cdot \cos \left(\theta_{i}, \theta_{S D}\right)$

Where $d_{N i D}$ represents the distance between node $N_{i}$ and $D$, $d_{S D}$ represents the distance between $S$ and $D . \gamma$ and $\delta$ represent score factors for the distance and direction respectively. The node $S$ would choose the neighbor with the best availability as the next-hop forwarder.

\section{PERFORMANCE EVALUATION}

We evaluate the performances of three protocols including GSR[4], GyTAR[7] and our proposed TAGR in NS-2.31 [13]. The urban area of Baotou, China is abstracted as the simulation environment with a $2 \mathrm{~km} \times 2 \mathrm{~km}$ area consists of 23 two-way roads. The total length of the roads is about 25 kilometers. In order to simulate vehicles' movement, we use the simulator SUMO [14]. It is an open source, microscopic traffic simulator that can model realistic mobility of vehicles. We build the road network, traffic signals according to digital map database and set random vehicle routes (a vehicle randomly moves to a destination with the shortest path) for the simulation area.

The vehicles move at a velocity from 30 to $80 \mathrm{~km} / \mathrm{h}$ and communicate using the IEEE 802.11DCF standard, with a 250 meters radio propagation range and 2 Mbps channel capacity. The simulation results are averaged over ten runs. Each takes 300 seconds of simulation time. 10 random connections are established using CBR at 1-10 packets per second and a packet size of 512 bytes. We further conduct simulations in three different vehicle densities: low, 200 nodes; high, 800 nodes.

\section{A. Packet delivery ratio}

Figure.2 shows that TAGR outperforms the other two protocols with highest delivery ratio for different CBR rates. This is mainly because that TAGR selects the entire forwarding path with the highest connectivity quality based on real-time traffic in the whole network. In addition, TAGR uses the movement adaptive method to choose next forwarder and consequently avoids link rupture. GSR performs poorly because the geographic shortest path often suffers from frequent network segmentation caused by uneven deployment of nodes. GyTAR obtains moderate performance, with the support of partial traffic information at intersections, higher than GSR but lower than TAGR.

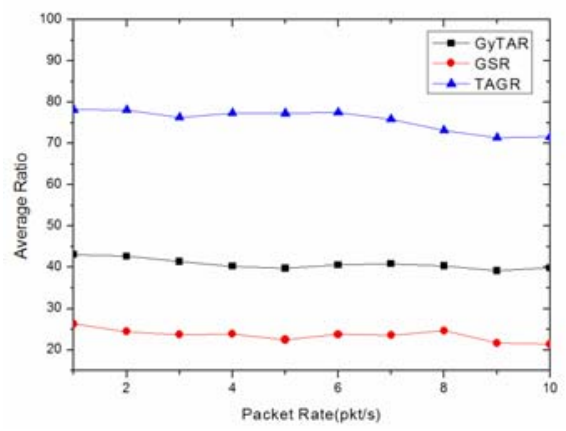

(a) Low Density - 200 nodes 


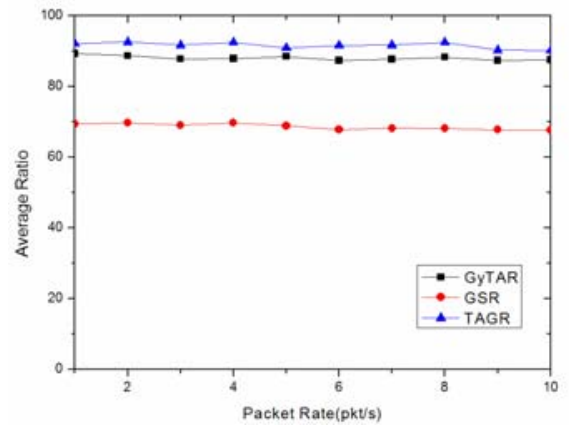

(b) High Density - 800 nodes

Fig 2. Packet Delivery Ratio

\section{B. Average End-to-End Delay}

As Figure 3 shows, the delay of TAGR is the lowest one in three protocols. The main reason that TAGR product a shorter delay is that, TAGR always select optimal path with denser traffic roads in advance and the packet forward process implements more wireless relay. However, GyTAR discoveries the route progressively when transfer data packets from source to destination. This will increase the chance of network partitions and more carry-and-forwards have to be conducted which prolong the average delay. For GSR, when a packet reaches a local maximum along its anchor path, GSR switches back to greedy mode. This would result in excessive hops as greedy forwarding does not perform well in a city environment.

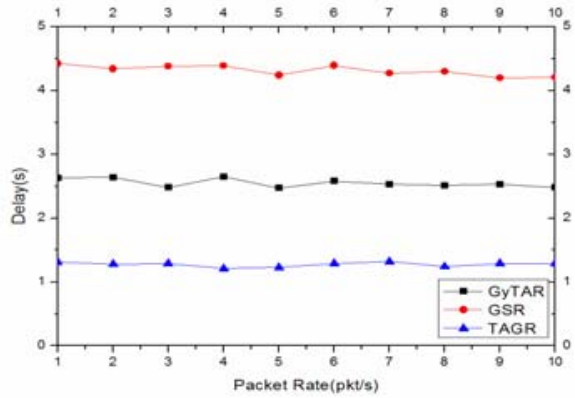

(a) Low Density - 200 nodes

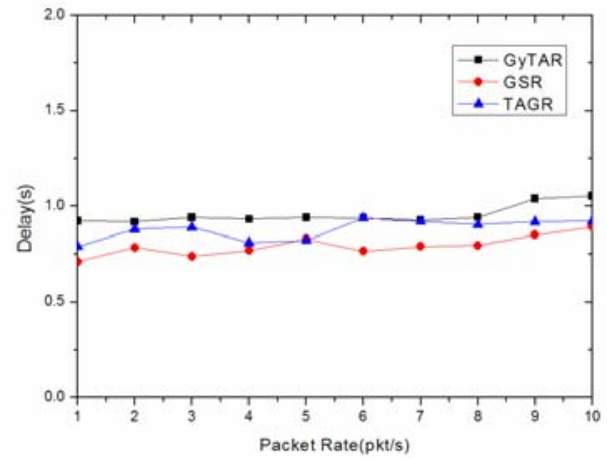

(b) High Density - 800 nodes

Fig 3. Average end-to-end delay

\section{Routing Overhead}

Fig. 4 shows the total routing overhead, measured in total number of routing packets sent per second. GyTAR generates the least because it only has the beacon messages. For GSR, the overhead consists mainly of the periodic beacons and the packets for the detour way in recovery mode. For MAGR, the overhead is presented as a cumulative contribution of beacon and constant packets for traffic estimation. Therefore, the overhead of GSR will be close to MAGR.

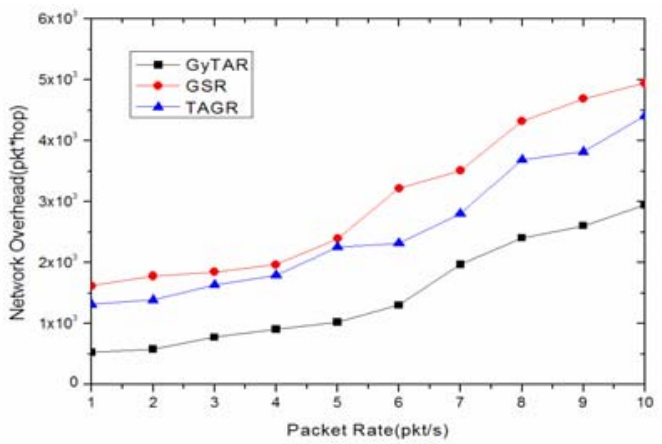

(a) Low Density - 200 nodes

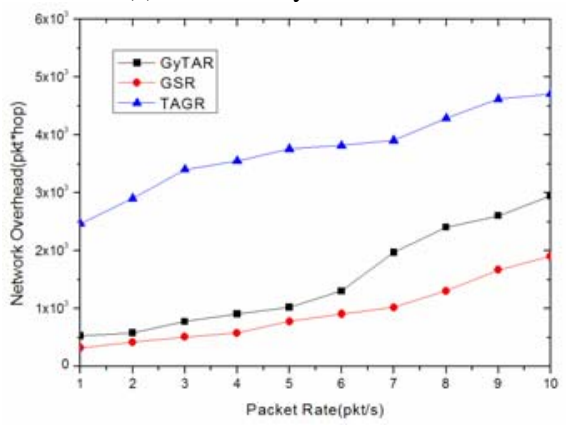

(b) High Density - 800 nodes

Fig 4. Routing Overhead

\section{CONCLUSION}

We propose a novel geographic routing scheme called TAGR for VANET in city environments. Following the location service that we have put forward, a real-time vehicular traffic estimation method is presented, which analyzes and aggregates location information to calculate vehicle densities of urban roads. Then, TAGR could use traffic information to create road-based paths with the best transmission quality. Moreover, an improved next-hop selection method based on movement predication is proposed for geographic packet forwarding between two successive intersections. In our comparative simulation study in NS2, TAGR outperforms the other two routing schemes in terms of packet delivery ratio and average end-to-end delay.

\section{REFERENCES}

[1] W. Kiess and M. Mauve, "A survey on real-world implementations of mobile ad-hoc networks," Elsevier Journal Ad Hoc Networks, 5(3), 2007.

[2] P. Li, P. Jannotti, and D.S.J. De Couto, etc, "A scalable location service for geographic ad hoc routing," In Proc. of ACM MobiCom, 2000.

[3] B Karp, H T Kung, "GPSR: Greedy perimeter stateless routing for wireless networks," In Proc. of ACM MobiCom , 2000.

[4] C Lochert, H Hartenstein, J Tian, Etc, "A routing strategy for vehicular ad hoc networks in city environments," In Proc. of IEEE IVS 2003. 
[5] J Zhao, G Gao, "VADD: Vehicle-Assisted Data Delivery in Vehicular Ad Hoc Networks," in Proc. of IEEE INFOCOM 2006.

[6] V Naumov, T-R Gross, "Connectivity-Aware Routing (CAR) in Vehicular Ad-hoc Networks," In Proc. of IEEE INFOCOM 2007.

[7] M Jerbi, S-M Senouci, R Meraihi, etc. "An Improved Vehicular Ad Hoc Routing Protocol for City Environments," In Proc. of IEEE ICC, 2007.

[8] J Nzouonta N Rajgure, G Wang, etc, "VANET Routing on City Roads Using Real-Time Vehicular Traffic Information," IEEE Transactions on Vehicular Technology, 2009, 58(7): 3609 - 3626.

[9] F Granelli, G Boato, and D Kliazovich, "MORA: A Movement-Based Routing Algorithm for Vehicle Ad Hoc Networks," in Proc. of IEEE GLOBECOM 2006.
[10] N Brahmi, M Boussedjra, J Mouzna, etc, "Adaptative Movement Aware Routing for Vehicular Ad Hoc Networks," in Proc. of ACM IWCMC 2009.

[11] S C Lo, W K Lu, "Design of Data Forwarding Strategies in Vehicular Ad Hoc Networks," in Proc. of IEEE VTC 2009 Spring.

[12] X Y Bai, X M Ye, J Li, H Jiang, "VLS: A Map-Based Vehicle Location Service for City Environments," In Proc. of IEEE ICC 2009.

[13] The Network Simulator: NS-2. http://www.isi.edu/nsnam/ns

[14] SUMO - Simulation of Urban Mobility.

http://sumo.sourceforge.net/links/simulations.shtm 